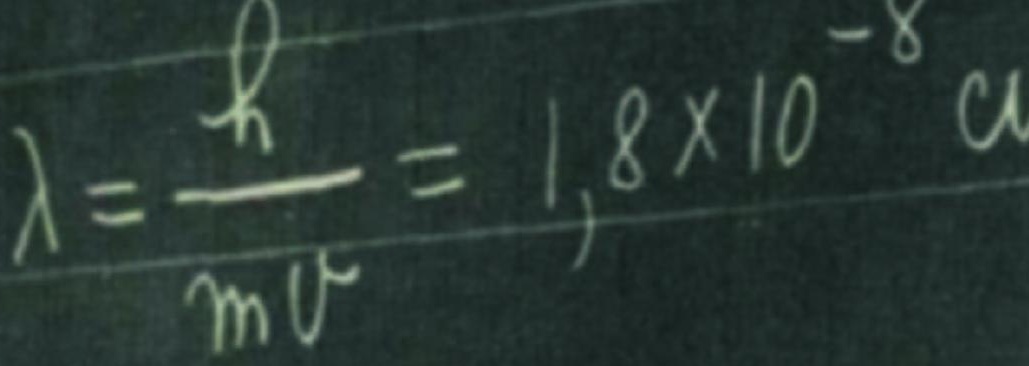

A TRIBUTE TO ENRICO FERMI

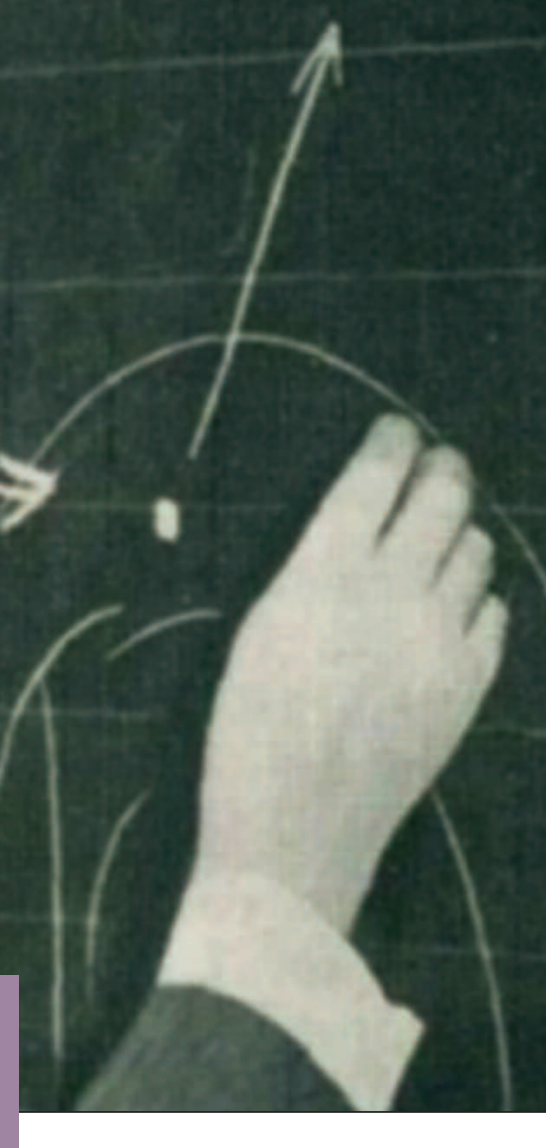

* Henk Kubbinga * University of Groningen (The Netherlands) * DOI: 10.1051/epn/2009803

In the history of physics, Italy has a place of its own. The hightimes of Galileo and Torricelli were followed by those of Galvani and Volta. But not much happened after 1830. Early in the $20^{\text {th }}$ century, though, the spirit of Galileo seemed to be reincarnated in Enrico Fermi (1901-1954).

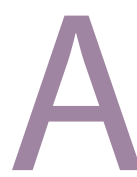

$s$ a youngster, Fermi attended the public schools of Rome, learned Latin and Greek, and had no trouble in reciting poems [1]. After the baccalaureat he enrolled at the Scuola Normale Superiore, at Pisa, to study mathematics and the language of science, i.e. German. The charming lad soon taught his teachers: as a second year's student he lectured on the quantum theory and the Bohr-Sommerfeld atom. Fermi's first post-doc trip abroad was to Göttingen, the contemporary Mecca of theoretical physics. There he worked with Max Born and the latter's students Werner Heisenberg and Pascual Jordan, and published on ergodicity and the adiabatic principle. These papers got translated into German and were noticed by Paul Ehrenfest, the renowned specialist in the field. As a result of a first contact, through George Uhlenbeck, Fermi visited Leiden in September-December 1924 and made the acquaintance of Lorentz,
Einstein, Kronig and Goudsmit. At the time the quantization of a 'gas' of equal particles had his particular attention. In 1926 this research gave way to his first major contribution to theoretical physics; it was on the 'Quantization of the ideal monoatomic gas.' The leading idea was to treat the translation of molecules between the parallel walls of a container as a periodic, and therefore quantizable, phenomenon. Instead of the container Fermi, next, posited a radial potential field around a central point - say, the origin of a Cartesian system of axes -, much like the Bohr-Sommerfeld atom. The monoatomic molecules were said to behave like harmonic oscillators of frequency $s_{i} v$ and to be subject to quantum numbers similar to those of the electrons, here $s_{1}, s_{2}$ and $s_{3}$, for the three axes, with $s_{i}=s_{1}+s_{2}+s_{3}$. The total energy $w$ of one such atom will thus be $s_{i} h v$. Apparently, an energy equal to 0 may only be realized in one way, an energy of $1 h v$ in three ways, etc. So the 
III position of lowest energy would only be open to one atom, the second lowest to three, etc., the degeneracy increasing with increasing number of atoms s. At $\mathrm{T}=0$ the gas would feature 1 atom with energy 0 , three with energy $1 h v$, etc. The $3 \mathrm{D}$-space around the center apparently corresponds to a lattice of cells of volume $h^{3}$, each cell ready to contain one atom. By taking, instead of $s_{1}$ $s_{2}$ and $s_{3}$, the three atomic quantum numbers, each with its own value scale, the same logic could be applied to the electrons in the Coulomb field of an atom's nucleus. Here are, of course, the roots of quantum electrodynamics.

\section{Bèta-decay; neutrinos and neutrons}

The research on radioactivity, particularly at Cambridge, Vienna, Paris and Berlin, had revealed that a-particles of a particular source had a characteristic energy: their traces in the Wilson-chamber were of uni-

V FIG. 1:

Enrico Fermi,

Werner Heisen-

berg and

Wolfgang Pauli at the Volta centennial at Lake Como (March 1927).

The photo was made by Franco Rasetti (courtesy of Emilio Segrè

Visual Archives of the American Institute of Physics). form length. By contrast, $\beta$-particles, supposed to pre-exist as electrons in the nuclei, were expelled with energies varying up to a certain maximum. Pauli had suggested, in 1930, the simultaneous escape of tiny neutral particles in order to maintain energy conservation. It concerned particles like Rutherford's neutrons but light as the electrons, endowed with an energy range that complemented the one of those electrons and for the rest unable to leave traces in the Wilson-chamber. Fermi c.s became involved simply by organizing a state-of-the-art conference in Rome, in October 1931. The next year was rich in new finds abroad: from Urey's deuteron (January) through Chadwick's neutron (February) to

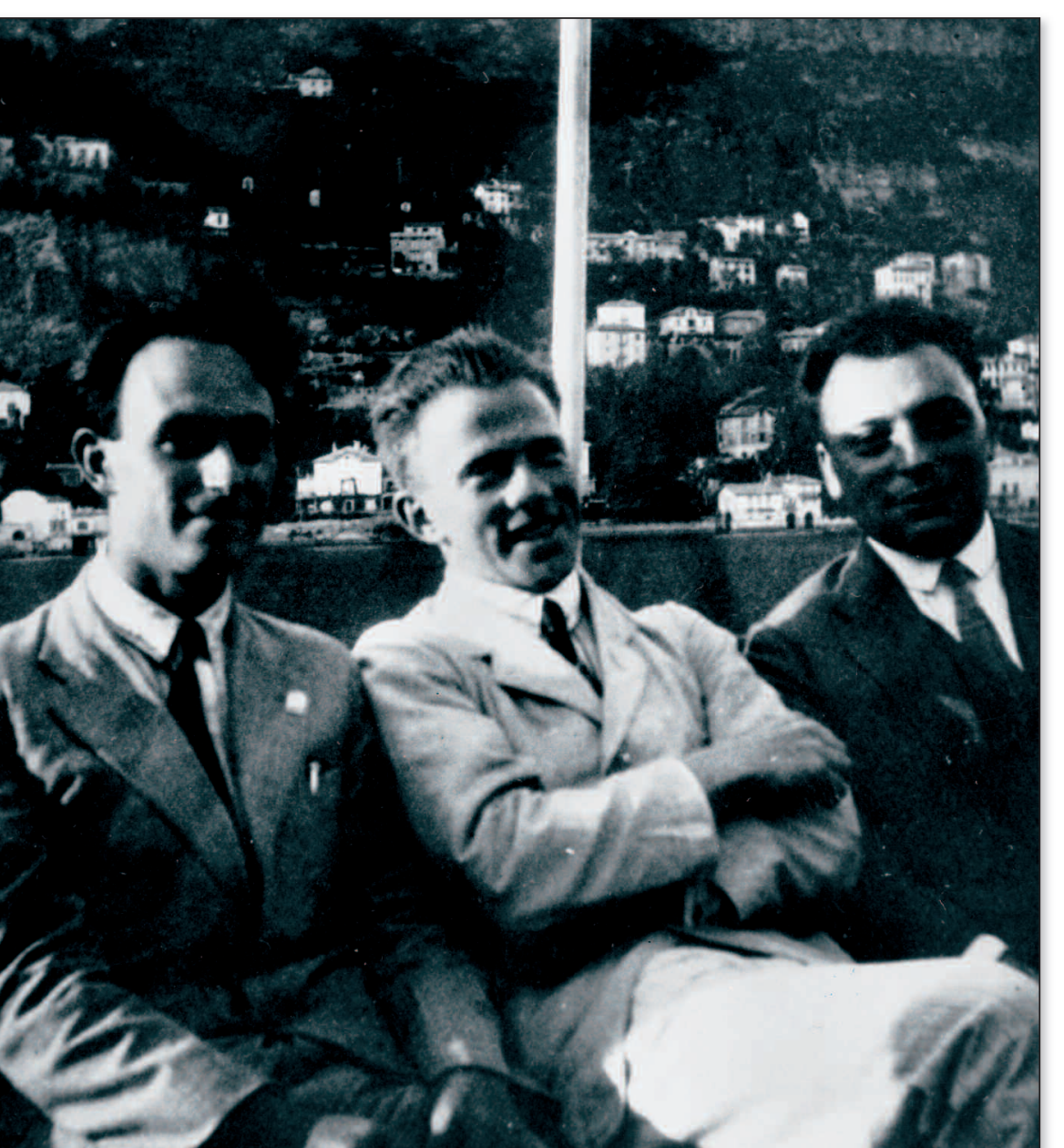

Anderson's positron (August). In the autumn of 1932 Fermi c.s. definitely started to focus on nuclear physics. Still without a programme, new instruments were designed, built and tested: a Wilson chamber, like Lise Meitner's, a gamma-ray single Bi-crystal spectrometer an ionization chamber, etc. As it happened 'The structure and properties of atomic nuclei became the theme of the next Solvay congress, that of October 1933; Fermi was among the key-notes. About this time Fermi himself elaborated on the precise nature of bèta-decay in a paper, that would become a classic in the field. The central idea was that electrons do not exist as such in the nucleus, but are produced in much the same way as photons during the decay of activated atomic states according to radiation theory. The difference between a proton and a neutron, then, is nothing but a difference in quantum states of one and the same nucleon: $\beta$-particles (and neutrinos) only exist between emission and absorption. Fields had to be quantized, such was the message. Eventual $\gamma$-radiation could be attributed to transitions between nuclear isomers.

When we may believe a newly (2004!) retraced lab notebook, it was March 1934 when Fermi planned experiments to bombard heavy elements with Chadwick's neutrons in order to test his own new theory of $\beta$-decay [2]. The principal piece was a steady neutron source, also of their own device. It was a radon-beryllium combination, the radon (halflife 3,8 days) stemming from Italy's national stock of radium. In a small glass bulb (length $2 \mathrm{~cm}$ ) containing already powdered $\mathrm{Be}$, the radon was concentrated with the help of liquid air. The Rn-Be-tube (50-500 mCurie) was put into the interior of a cylindrical sample of platinum. Why platinum? Its large excess of neutrons might have been the reason, perhaps together with its ready availability. An extra neutron could possibly trigger $\beta$-decay. However, Pt did not show any effect at all. Aluminium - a pill tube? - happened to be tested next. Aluminium had been a hot topic on its own account since the Joliot-Curies had established, a few months earlier, that polonium's $\alpha$-rays turned it into phosphorus, the new radioactive species emitting positive electrons, those detected by Anderson in cosmic rays. This time Fermi's counter revealed a strong activity, suggestive of the transformation of ${ }_{13} \mathrm{Al}^{27}$ into ${ }_{11} \mathrm{Na}^{24}$ under $\alpha$-emission, followed by $\beta$-decay resulting in the stable isotope ${ }_{12} \mathrm{Mg}^{24}$. His report, initially in Italian, was dated 25 March 1934. All the elements at his disposal were checked in a hurry. Some of the lighter elements were tested in the form of paraffin and water: $\mathrm{H}, \mathrm{C}$ and $\mathrm{O}$ did not give appreciable effects, though.

Fermi's thrilling finds were warmly received by the experimentalists, particularly by the most distinguished among them, Ernest Rutherford, Cambridge. On 10 May 1934 the team reported also on uranium. 
When $U$ was separated from its $\beta$-active products and subsequently bombarded with neutrons, several reactions showed up, among which a $\beta$-decay with a half-time of 13 minutes. Fermi c.s. had the impression that a new species was at issue, an element with atomic number 93: ${ }_{92} \mathrm{U}^{238}+\mathrm{n}^{1} \rightarrow{ }_{92} \mathrm{U}^{239} \rightarrow{ }_{93} \mathrm{X}^{239}+1$ e. The new element was provisionally baptized 'ausonium', a possibly implied element number 94 'hesperium', discrete references to Rome and Italy, respectively. The new elements, though, were short-lived: in December 1938 the quartet Hahn, Strassmann, Meitner and Frisch showed that it had been a matter of 'fission'.

\section{Slow neutrons}

After the summer holidays, in mid-September 1934, Fermi's team planned to standardize the procedures and establish a semi-quantitative scale of the induced activities, starting with silver. Accidentally, they noticed that when the Ag cylinder was irradiated on a wooden table, the effect was far larger, than on the marble table in the same room. The material of the table, apparently, played a role. Paraffin wax, already investigated before by the Joliot-Curies and Chadwick - and by Fermi himself, as we saw above -, was now used as an analog of wood. October 20, 1934, a Saturday, Fermi, irradiated the silver cylinder through a layer of paraffin, hurried it to a counter and noticed an increase in activity by a factor of about 100. A 'filter' of pure carbon did not have any effect, so it seemed that it was the hydrogens that did the job. Fermi realized that neutrons traveling through paraffin wax regularly hit hydrogen nuclei of about the same mass, inelastic collisions that caused them to slow down. The apparently fast neutrons escaping from the source were turned into slow ones with, in the end, thermal velocities (2600 m.sec ${ }^{-1}$ ); these thermal neutrons were, quite unexpectedly, more readily absorbed by silver nuclei. Among the tested metals was also cadmium: it was the exception in that it only absorbed neutrons under $\gamma$-emission, but did not feature $\beta$-decay.

One of the experiments concerned a plate of rhodium, the most sensitive metal, on top of a paraffin cylinder, just above a Rn-Be-tube (Figure 2). When a hat-like other paraffin cylinder was put over the Rh plate, the latter's activity increased. It seemed as if the slow neutrons diffused also into the upper part, were scattered around in the wax and eventually, on escaping, hit the Rh plate also from above. A similar effect was noticed when the Rh was covered with a layer of beryllium, carbon or silicon: these layers behaved like mirrors, causing a back-scattering of neutrons that had passed the Rh plate. Boron did not work. On the other hand, some materials had a decreasing effect when brought as a layer inbetween the rhodium plate and the neutron source, particularly yttrium, cadmium and, indeed, the boron that didn't work on top of the rhodium. For Cd, for

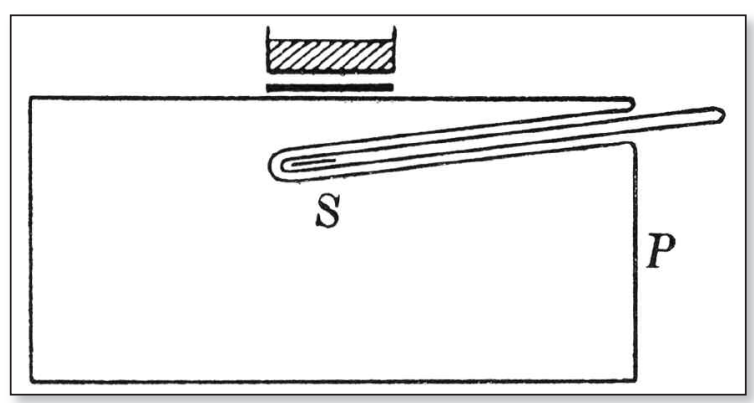

instance, the half-value thickness of such a layer was 0.014 g.cm ${ }^{-2}$, corresponding to a nuclear cross-section of $10,000 \times 10^{-24} \mathrm{~cm}^{2}$. The neutrons were absorbed, not scattered, by these materials, so much was sure.

Already in 1935 Fermi was nominated for the Nobel Prize for Physics. In 1938 a crescendo of proposals finally resulted in his being awarded the prize "for his demonstrations of the existence of new radioactive elements produced by neutron irradiation and for his related discovery of nuclear reactions brought about by slow neutrons". With his wife Laura and their children he left afterwards for Columbia University, New York, for a six months stay which turned into emigration.

If Fermi was generally considered the 'Pope' of physics, his team functioned as a true Curia. It was the time that men like Max Planck and Niels Bohr were among those elected member of Rome's newly founded Pontifical Academy of Sciences. Fermi, then, had no problems in acknowledging, on occasion, his great debt to the curator of Italy's stock of radiumchloride, Giulio Cesare Trabacchi: indeed, what would a Pope do without whom he called his 'Divine Providence'?

\section{Acknowledgment}

The author is indebted to the Società Italiana di Fisica (Bologna) and the Reale Scuolà Normale Superiore (Pisa) for kind help and gladly acknowledges the hospitality of the Accademià Nazionale dei Lincei (Rome) and the Department of Physics of the University of Rome.

\section{About the author}

Henk Kubbinga (University of Groningen) is a member of the EPS-History of Physics Group. With some delay his book The molecularization of the world picture, or the rise of the Universum Arausiacum has finally appeared in print (Groningen University Press, 2009).

\section{References}

[1] For Fermi's work, see Collected papers - Note e memorie (Chicago: University of Chicago Press; Rome: Accademià Nazionale dei Lincei, 1962). For appraisals, cf. C. Bernardini and L. Bonolis (eds.), Enrico Fermi: his work and legacy (Bologna: Società Italiana di Fisica; Berlin etc.: Springer Verlag, 2004).

[2] Cf. F. Guerra et al., Physics in perspective 8 (2006) 255-281. We follow their analysis.
4FIG. 2:

A paraffin wax cylinder P containing a hole for the $\mathrm{Rn}-\mathrm{Be}$ source $S$ (kept in a long tube). The materials to be studied were put on top. One of the most active was rhodium; it was used as a detector to distinguish 'scattering' from 'absorption'. Here it carries a glass dish, which was filled e.g. with boric acid solutions to study boron's effect. 